\title{
Engineering kidneys from simple cell suspensions: an exercise in self-organization
}

\author{
Jamie A. Davies • C-Hong Chang
}

Received: 28 May 2013 /Revised: 28 June 2013 / Accepted: 12 July 2013 / Published online: 30 August 2013

(C) The Author(s) 2013. This article is published with open access at Springerlink.com

\begin{abstract}
Increasing numbers of people approaching and living with end-stage renal disease and failure of the supply of transplantable kidneys to keep pace has created an urgent need for alternative sources of new organs. One possibility is tissue engineering of new organs from stem cells. Adult kidneys are arguably too large and anatomically complex for direct construction, but engineering immature kidneys, transplanting them, and allowing them to mature within the host may be more feasible. In this review, we describe a technique that begins with a suspension of renogenic stem cells and promotes these cells' self-organization into organ rudiments very similar to foetal kidneys, with a collecting duct tree, nephrons, corticomedullary zonation and extended loops of Henle. The engineered rudiments vascularize when transplanted to appropriate vessel-rich sites in bird eggs or adult animals, and show preliminary evidence for physiological function. We hope that this approach might one day be the basis of a clinically useful technique for renal replacement therapy.
\end{abstract}

Keywords Tissue engineering - Adaptive self-organization • Transplantation $\cdot$ Angiogenesis $\cdot$ Organ culture

\section{Clinical need for renal tissue engineering}

Renal disease is a serious and increasing problem all over the developed world. A combination of congenital kidney diseases, acute chemical damage (including pharmacological), infection and damage from chronic diseases such as diabetes and idiopathic hypertension mean that a large number of people reach end-stage renal disease (ESRD) [1]. Two common treatments exist: dialysis to maintain life and, for more

J. A. Davies $(\bowtie) \cdot$ C.-H. Chang

University of Edinburgh, Edinburgh, Scotland, UK

e-mail: jamie.davies@ed.ac.uk fortunate patients, transplantation of a functional kidney. There is, however, a serious shortage of transplantable kidneys: the waiting list is around 7,000 people in the UK alone. This creates an urgent need for new developments in preventing renal damage in the first place, in regenerating damaged kidney tissue in situ and in engineering new transplantable kidneys when endogenous kidney function cannot be saved. This review focuses on the last of these needs.

A full-scale adult kidney is an anatomically complicated structure that contains $>60$ cell types [2]. Engineering a new one is therefore unlikely to be easy. Different research groups currently take one of three broad approaches: (1) direct construction by printing, (2) repopulation of decellularised matrix and (3) construction of simple kidney progenitors intended to mature in a host. So far, although the idea of printing a kidney has been talked about within the research community and in more public platforms [3], the spatial resolution and cellular complexity required is beyond existing technologies. Repopulating a decellularised matrix is more promising. It still requires a decellularised donor kidney, but in a postmortem condition, this could be far worse than the minimum required for living transplant. It also requires a source of adult kidney cells to perform the repopulation, so is not exactly a solution to transplant waiting lists, although conceivably a patient's own remaining cells from a damaged kidney may be persuaded to multiply enough to colonize the matrix of a good one. So far, the best experiments with decellularisation have succeeded in at least limited repopulation and better filtration than achieved by decellularised matrix alone [4]. There is still a long way to go from here to acceptable functionality.

The idea of using stem cells to engineer 'foetal kidneys' that can be grafted into a host to grow and mature in situ is the only one of these approaches that needs neither cells nor matrix from an adult kidney. Therefore, it might in principle be a way of escaping the restrictions of waiting lists. This method, too, has a very long way to go before it is clinically 
practical, but this approach is the subject of the rest of this review.

\section{Overview of the engineering-then-maturation approach}

There are essentially three steps to the 'engineering-thenmaturation' approach (Fig. 1). The first is to generate renogenic (kidney-producing) stem cells from a patient's own induced pluripotent stem cells (iPS) cells or some other convenient stem cell source. The second is to engineer a foetal kidney from the simple suspension of stem cells that would be obtained from cultures. The third is to graft the engineered rudiment in a way that promotes its maturation and function. Each of these stages presents quite different problems and requires a different set of skills.

The first problem, that of differentiating embryonic (ES) or iPS cells into renogenic stem cells is often called reprogramming, although the term is poorly chosen because the aim is not to alter the natural developmental programme in the genome, but, rather to move cells to the kidney-producing part of it. Several groups have made progress with this: One approach uses specific exogenous growth factors to mimic the succession of signals that cells would receive in their progress from inner cell mass via mesoderm and intermediate mesoderm to metanephrogenic mesoderm or ureteric bud [5]. Another approach uses culture in a niche. A third approach works by directly transfecting the cells with appropriate transcription factors [6]. Each of these approaches remains a work in progress, but production of stem cells with some apparently renogenic characteristics has been achieved, albeit with low efficiency $[6,7]$.

Even when the problem of making renogenic stem cells has been solved, a culture of stem cells is not a kidney. A critical step is therefore the development of a technique that enables tissue engineering a small, properly organised kidney. Our work has been directed towards this step, and a technique for performing it is the main focus of the rest of this review. Before discussing this specific step in detail, however, it is worth considering the feasibility of 'downstream' techniques for transplantation of the engineered foetal kidneys into adult hosts. Experiments on grafting embryonic kidneys into adults began in the 1940s and included grafting to the omentum [8], into the eye [9, 10] and subcutaneously [11]. In rats, E15 foetal kidneys transplanted into uninephrectomised or pregnant adult hosts grew and became vascularised; between 17 and 21 days later, it was possible to connect their ureters to that of the host [12]. As these foetal kidneys continued to mature, they produced urine, although with only about $6 \%$ of normal glomerular filtration rate [13]: for comparison, haemodialysis of humans provides the equivalent of about $10 \%$ normal filtration rate. If the remaining adult kidney was removed, the transplants functioned well enough to produce a modest prolongation of host life - not enough to be clinically useful, but perhaps enough to indicate that these existing experiments provide a foundation on which better techniques might be constructed [13].

The hope of the engineering-then-maturation approach is that the three critical steps, each currently being tackled almost in isolation from the other two, can be refined and then connected to complete a pathway from stem cells to function$\mathrm{al}$, transplanted, engineered rudiment.

\section{Development of a renal tissue engineering technique: the stem cell reaggregation approach}

Embryonic kidneys develop almost autonomously: even when removed from the rest of the embryo and cultured in simple media, intact organ rudiments will develop essentially organotypically (except for a lack of blood vessels) to produce nephrons arranged around and connected to a branched collecting duct system $[14,15]$. When first isolated from the embryo, the organ rudiments consist of two stem cell typesureteric bud and metanephrogenic mesenchyme - which will each later produce further cell types. Clearly, these cells and the communication loops between them [16] are capable of sufficient adaptive self-organisation [17] to arrange them-

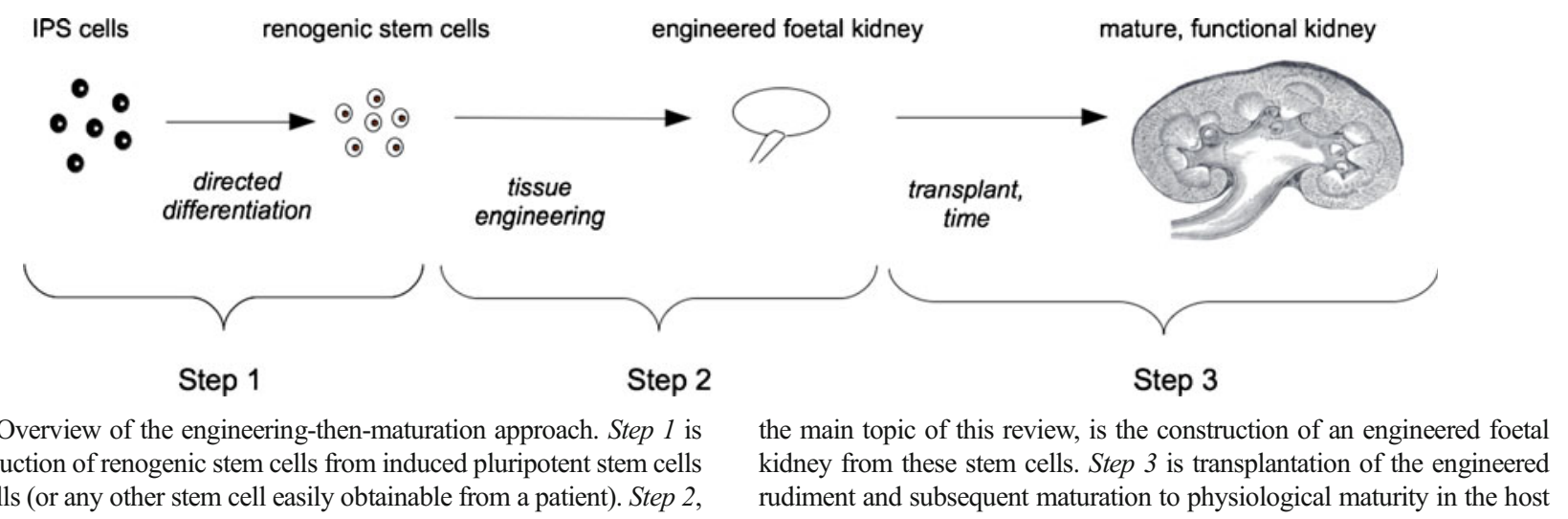


selves into a foetal organ without detailed external signals and even to make an appropriately unusually shaped organ to fit an unusually constrained environment. A trivial example of this plasticity, familiar to many in the field, is the ability of kidneys to grow flat on a filter instead of three-dimensionally in an embryo. Coming from a research background with a theoretical interest in self-organising systems, we reasoned from known communication loops that the self-organising ability of the cells may be sufficiently high to work even when their original anatomical relationships have been erased. This is the abstract basis of the stem cell reaggregation approach.

Because there is so far no reliable way of producing significant numbers of renogenic stem cells as pure populations from ES, iPS or mesenchymal stem cells (MSCs), we began by harvesting E10.5 mouse kidneys and dissociating them into simple cell suspensions. These suspensions are a simulation of what we hope will, one day, be available from a reliable iPS-to-renogenic stem cell process: it has never been our intention to engineer human kidneys for clinical use from suspensions of human fetal cells for obvious ethical reasons.

Simply bringing suspensions of mixed ureteric bud and metanephrogenic mesenchymal cells together by centrifuging them into a random aggregate does not result in development of renal structures: instead, the cells die. This cell death is not surprising: suspending the cells removes them from their natural cell-cell and cell-matrix contacts. In general, when untransformed cells lose these contacts, they die by a type of programmed cell death called anoikis [18], probably a valuable safety mechanism for eliminating cells that are erroneously placed during natural development. A quite different line of research in the laboratory, on the role of the cytoskeleton in ureteric bud branching [19], indicated that inhibiting the rho-dependent-kinase (ROCK) strongly inhibited elective cell death that is a normal feature of metanephric development
[20]. Incubation of reaggregated suspensions of renogenic stem cells with ROCK-inhibiting drugs suppressed anoikis and promoted survival but interfered dramatically with subsequent morphogenesis, as might be expected from the effects of ROCK inhibition on intact kidneys [19]. An improved technique, which used ROCK-inhibiting drugs to protect reaggregates for their first $24 \mathrm{~h}$ and then replaced them with simple media, was much more successful [21]. Over the next few days, the reaggregates formed many small, branching ureteric bud tree-lets, which induced early nephron formation in the surrounding mesenchyme. By morphology and gene expression, the development of these nephrons was normal, and they connected to the ureteric bud tree-lets (Fig. 2a).

\section{Arranging the kidney around one collecting duct system}

The problem with the simple reaggregation system described above is that it produces many small collecting duct tree-lets rather than a single connected tree that could be joined to a ureter. Clearly, a kidney made in this way, even if it could be connected to the blood system, would not be useful because the filtrate would have nowhere to go. Also, at a more subtle level, there would be no clear corticomedullary organisation. Formation of multiple small collecting duct tree-lets is inherent in the manner in which the system is assumed to work, as ureteric bud cells aggregate together to make multiple small clumps that cannot be expected to join because growing ureteric bud branches show mutual repulsion (Davies et al., unpublished data).

A method by which to engineer kidneys arranged around a single collecting duct system was developed by us [22] using a serial culture method. The first step is a basic reaggregation culture to produce a 'kidney' with many small ureteric bud

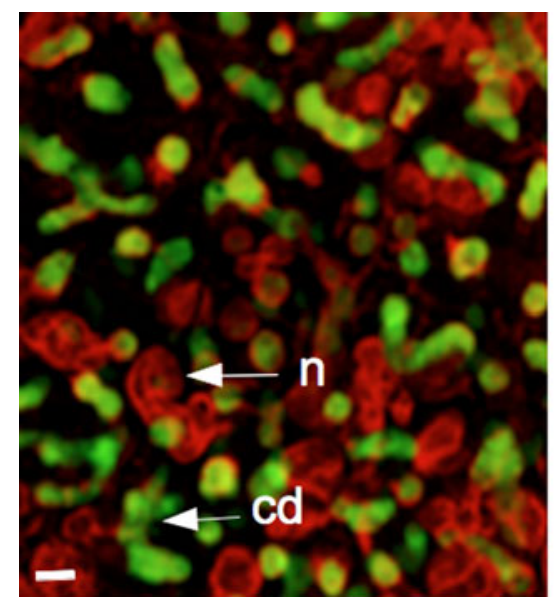

Fig. 2 Engineered renal tissues produced by single (left panel) and serial (right panel) reaggregation. Each contains nephrons ( $n$ : red) and collecting ducts ( $c d$ : green); in the single reaggregation system, the organ is disorganised because there are many collecting duct tree-lets rather than

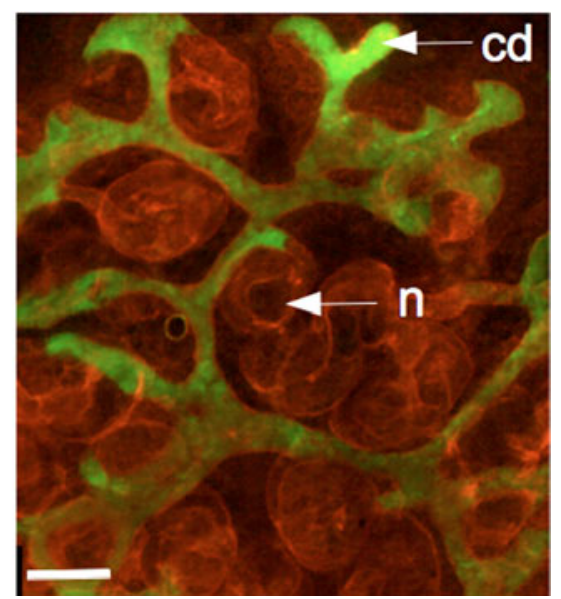

one tree. The serial reaggregation system generates a kidney organised around a single collecting duct tree. These micrographs are 'spare' images from data sets described in our earlier publications [21, 22] 
tree-lets, as above. One of these is then isolated and surrounded by a fresh suspension of metanephrogenic mesenchymal cells. The result is ramification of the tree-let into a single collecting duct tree and conversion of metanephrogenic mesenchymal cells into cap mesenchyme, nephrons and stroma in the usual manner, with nephrons connecting to the collecting duct system (Fig. 2b). Under appropriate culture conditions that favour corticomedullary zonation in intact kidney rudiments, these serial reaggregates show a distinct outer cortex and inner medulla and medullary extension of loops of Henle [23].

\section{Connecting to a blood supply}

Reaggregated kidneys, as with intact kidney rudiments cultured in incubators filled with $5 \%$ carbon dioxide $\left(\mathrm{CO}_{2}\right)$ in air, show little or no development of a vascular system. This is a problem for tissue engineering for the purposes of regenerative medicine, the main physiological function of a kidney is to filter blood: normal adult human kidneys receive about $20 \%$ of cardiac output. Clearly, some way has to be found to connect the nephrons of engineered kidneys to flowing blood.

The metanephrogenic mesenchyme of kidney rudiments contains endogenous endothelial progenitors even though they do not differentiate well in normal culture. Endothelial differentiation can be encouraged by incubation in very low oxygen, but this results in large masses of endothelial cells rather than organised vessels [24]. If a kidney rudiment is cultured with an external source of vessels that are capable of angiogenesis, for example the chorioallantoic membrane (CAM) of a bird egg, it is capable of attracting exogenous blood vessel branches and thereby gaining a blood supply [25, 26]. When such grafts are made from one strain or species into another, it is possible to determine that blood vessels are in fact derived from both graft and host, generally the larger vessels being host-derived and the smaller ones coming from the graft's own mesenchyme [27]. Presumably, the incoming exogenous vessels somehow stimulate differentiation of endogenous endothelial progenitors, though the biology of this is not understood.

Whatever the underlying organising mechanisms, the ability of kidney rudiments to acquire a vascular system when placed near host vessels offers a possible method for equipping cell-derived reaggregate kidneys with a functional blood supply. Grafting them on chick CAM (Fig. 3a) results in ingrowth of host vessels (Fig. 3b) and formation of glomerulus-type structures in the graft (Fig. 3c).

\section{First functional tests}

It has been known for some years that foetal kidneys transplanted into adult hosts can organise a blood supply, grow and show some physiological activity [13]. Logically, if the engineered foetal kidneys really are like their natural counterparts, they should behave the same way. We adapted the methods used in foetal kidney transplantations to assess the ability of engineered reaggregated kidneys to function in a mammalian host. Integration with the host vasculature happened efficiently only when the reaggregates were presoaked in vascular endothelial growth factor (VEGF), a known stimulant of angiogenesis [28]. When VEGF was used, however, host vessels entered the grafted engineered kidney. Injected fluorescently labelled albumen crossed from the blood system to enter the urinary space and was recovered by proximal tubule cells [29]. On a positive note, this implies that fluid flow from vessels to urinary space is taking place, presumably through glomeruli, and that at least some proximal reuptake function is normal. On the other hand, a well-constructed glomerulus should not allow major flow of molecules as large as albumin across it. In the system used, quantitative measurement was not possible, so the physiological quality of the engineered, grafted kidneys remains unclear with respect to filtration, recovery or concentration.
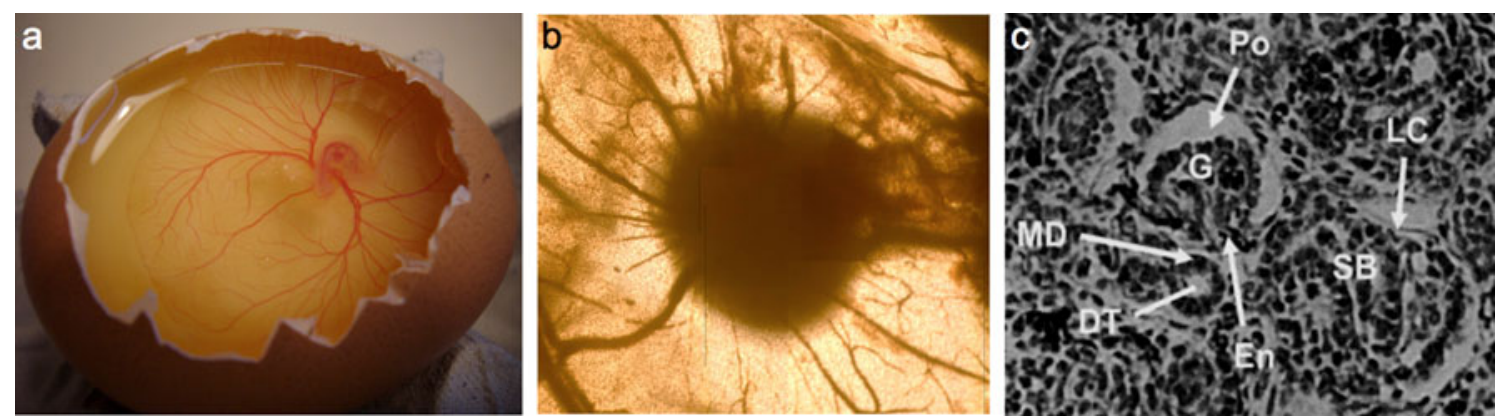

Fig. 3 Vascularisation of engineered kidney rudiments on chick chorioallantoic membrane $(C A M)$. a The CAM itself. b Chick vessels entering an engineered kidney rudiment (the dark object, centre). c A section through such a kidney: present are S-shaped body-stage $(S B)$ nephrons and more mature nephrons that have Bowman's capsules with glomeruli $(G)$ and a lower cleft $(L C)$ consisting of podocytes $(P o)$ and endothelial cells $(E n)$. As in normal nephrons, the distal tubule $(D T)$ approaches the glomerular entrance, where it makes a macula densa $(M D)$ 


\section{Prospects}

From the point of view of tissue engineering with a view to transplantation, the most pressing needs are to: (1) investigate how much engineered kidneys can grow and mature after grafting, (2) establish the physiological parameters of the engineered kidneys and (3) find a way of obtaining renogenic stem cells from a patient (for example, by partly differentiating iPS cells).

Given the similarity between engineered foetal kidneys and real ones, existing programmes of research and development on transplantation of foetal kidneys might be expected to improve the prospects for transplanted kidneys, even if such programmes are not specifically aimed at doing this. There are already some encouraging results from transplantation of foetal kidneys to large animals instead of rodents. E28 pig kidney to adult pig transplants, for example, show growth and production of new nephrons while in the host [30,31]. Likely areas for development include finding better sites for engraftment, protection of immature kidneys from adult blood pressure (something likely to be a problem in humans, even if it is not in rodents), and possibly developing ways to engraft many kidneys into one host so they can share the physiological load. Identifying humoral changes that take place on unilateral nephrectomy and during pregnancy, both of which are already known to encourage growth of grafted kidney rudiments, may also be useful.

Testing the physiological function of engineered kidneys is difficult in the context of a mammalian host. It may be better to return to the chick CAM system, in which the kidney is plumbed in to a pumped blood supply powered by the chick embryo heart. This system is highly accessible for injection of tracer molecules or drugs into the blood supply and to sampling from the collecting duct system. This would allow measurement of filtration rates, cutoff, concentration of filtrate, osmolarity of the medullary stroma etc.

The problem of obtaining renogenic stem cells may turn out to be the most frustrating, or it may fall quickly. The reaggregate system offers a highly valuable tool for stem cell biologists because it provides a clear test for renogenic activity. In particular, it is possible to mix marked candidate stem cells with the embryo-derived kidney stem cells and then to test whether they contribute to making a chimeric engineered organ [20,32]. This has already been used to test the abilities of some suspected stem cells with results that are, frankly, disappointing compared with the abilities of cells straight from the kidney-forming part of the embryo [33-36]. Any cells that do pass this test would be highly promising candidates for practical use.

The entire approach of tissue engineering as a means of restoring renal function will not be helpful in all patients with ESRD: it will probably be most suited to diseases involving gradual but unstoppable renal degeneration, which allow time for maturation of a graft in a still healthy body rather than for fast-onset, acute failure. Even if it can be made to work as well as hoped, engineering new organs will probably be just one technique amongst many, including conventional transplantation and, hopefully, stem-cell-based regeneration in situ.

Conflict of Interest The authors declare that they have no personal financial stake in this work or any other conflicting interests.

Open Access This article is distributed under the terms of the Creative Commons Attribution License which permits any use, distribution, and reproduction in any medium, provided the original author(s) and the source are credited.

\section{References}

1. Steenkamp R, Castledine C, Feest T, Fogarty D (2011) UK renal registry 13th annual report (December 2010): Chapter 2: UK RRT prevalence in 2009: National and centre-specific analyses. Nephron Clin Pract 119(Suppl 2):c27-52

2. Little MH, Brennan J, Georgas K, Davies JA, Davidson DR, Baldock RA, Beverdam A, Bertram JF, Capel B, Chiu HS, Clements D, Cullen-McEwen L, Fleming J, Gilbert T, Herzlinger D, Houghton D, Kaufman MH, Kleymenova E, Koopman PA, Lewis AG, McMahon AP, Mendelsohn CL, Mitchell EK, Rumballe BA, Sweeney DE, Valerius MT, Yamada G, Yang Y, Yu J (2007) A high-resolution anatomical ontology of the developing murine genitourinary tract. Gene Expr Patterns 7:680-699

3. Atala A (2010) Growing New Organs. TED lecture available at https://www.ted.com/talks/anthony_atala_growing_organs_engineering tissue.html

4. Song JJ, Guyette JP, Gilpin SE, Gonzalez G, Vacanti JP, Ott HC (2013) Regeneration and experimental orthotopic transplantation of a bioengineered kidney. Nat Med. doi:10.1038/nm.3154

5. Kim D, Dressler GR (2005) Nephrogenic factors promote differentiation of mouse embryonic stem cells into renal epithelia. J Am Soc Nephrol 16(12):3527-3534

6. Hendry CE, Little MH (2012) Reprogramming the kidney: A novel approach for regeneration. Kidney Int 82(2):138-146

7. Harari-Steinberg O, Pleniceanu O, Dekel B (2011) Selecting the optimal cell for kidney regeneration: Fetal, adult or reprogrammed stem cells. Organogenesis 7(2):123-134

8. Waterman AJ (1940) Growth and differentiation of kidney tissue of the rabbit embryo in omental grafts. J Morphol 67:369-385

9. Runner MN (1946) The development of the mesonephros of the albino rat in intraocular grafts. J Exp Zool 103:305-319

10. Grobstein C, Parker G (1958) Epithelial tubule formation by mouse metanephrogenic mesenchyme transplanted in vivo. J Nat Cancer Inst 2:107-119

11. Barakat TI, Harrison RG (1971) The capacity of fetal and neonatal renal tissues to regenerate and differentiate in a heterotopic allogenic subcutaneous tissue site in the rat. J Anat 110:393-407

12. Hammerman MR (2007) Transplantation of renal primordia: renal organogenesis. Pediatr Nephrol 22(12):1991-1998

13. Rogers SA, Lowell JA, Hammerman NA, Hammerman MR (1998) Transplantation of developing metanephroi into adult rats. Kidney Int 54:27-37

14. Rienhoff WF (1922) Development and growth of the metanephros or permanent kidney in chick embryos. Johns Hopkins Hosp Bull 33:392-406

15. Grobstein C (1953) Inductive epithelio-mesenchymal interaction in cultured organ rudiments of the mouse. Science 118:52-55 
16. Davies JA, Fisher CE (2002) Genes and proteins in renal development. Exp Nephrol 10:102-113

17. Davies JA (2013) Mechanisms of morphogenesis, 2nd edn. Elsevier Academic Press, London

18. Frisch SM (2000) Anoikis. Methods Enzymol 322:472-479

19. Michael L, Sweeney DE, Davies JA (2005) A role for microfilamentbased contraction in branching morphogenesis of the ureteric bud. Kidney Int 68(5):2010-2018

20. Coles HS, Burne JF, Raff MC (1993) Large-scale normal cell death in the developing rat kidney and its reduction by epidermal growth factor. Development 118(3):777-784

21. Unbekandt M, Davies JA (2010) Dissociation of embryonic kidneys followed by reaggregation allows the formation of renal tissues. Kidney Int 77(5):407-416

22. Ganeva V, Unbekandt M, Davies JA (2011) An improved kidney dissociation and reaggregation culture system results in nephrons arranged organotypically around a single collecting duct system. Organogenesis 7(2):83-87

23. Chang CH, Davies JA (2012) An improved method of renal tissue engineering, by combining renal dissociation and reaggregation with a low-volume culture technique, results in development of engineered kidneys complete with loops of henle. Nephron Exp Nephrol 21(34):e79-85

24. Loughna S, Yuan HT, Woolf AS (1998) Effects of oxygen on vascular patterning in Tie1/LacZ metanephric kidneys in vitro. Biochem Biophys Res Commun 18;247(2):361-366

25. Atterbury RR (1923) Development of the metanephric anlage of chick in allantoic grafts. Am J Anat 31:409-436

26. Sariola H, Timpl R, von der Mark K, Mayne R, Fitch JM, Linsenmayer TF, Ekblom P (1984) Dual origin of glomerular basement membrane. Dev Biol 101(1):86-96
27. Woolf AS (1998) Loughna S. Origin of glomerular capillaries: Is the verdict in? Exp Nephrol 6:17-21

28. Ferrara N (2009) VEGF-A: A critical regulator of blood vessel growth. Eur Cytokine Netw 20(4):158-163

29. Xinaris C, Benedetti V, Rizzo P, Abbate M, Corna D, Azzollini N, Conti S, Unbekandt M, Davies JA, Morigi M, Benigni A, Remuzzi G (2012) In vivo maturation of functional renal organoids formed from embryonic cell suspensions. J Am Soc Nephrol 23(11):1857-1868

30. Rogers SA, Talcott M, Hammerman MR (2003) Transplantation of pig metanephroi. ASAIO J 49:48-52

31. Hammerman MR (2009) Xenotransplantation of pancreatic and kidney primordia-where do we stand? Transpl Immunol 21(2):93-100

32. Davies JA, Unbekandt M, Ineson J, Lusis M, Little MH (2012) Dissociation of embryonic kidney followed by reaggregation as a method for chimeric analysis. Methods Mol Biol 886:135-146

33. Siegel N, Rosner M, Unbekandt M, Fuchs C, Slabina N, Dolznig H, Davies JA, Lubec G, Hengstschläger M (2010) Contribution of human amniotic fluid stem cells to renal tissue formation depends on mTOR. Hum Mol Genet 19(17):3320-3331

34. Kuzma-Kuzniarska M, Rak-Raszewska A, Kenny S, Edgar D, Wilm B, Fuente Mora C, Davies JA, Murray P (2012) Integration potential of mouse and human bone marrow-derived mesenchymal stem cells. Differentiation 83(3):128-137

35. Rak-Raszewska A, Wilm B, Edgar D, Kenny S, Woolf AS, Murray P (2012) Development of embryonic stem cells in recombinant kidneys. Organogenesis 8(4):124-136

36. Ranghini E, Mora CF, Edgar D, Kenny SE, Murray P, Wilm B (2013) Stem cells derived from neonatal mouse kidney generate functional proximal tubule-like cells and integrate into developing nephrons in vitro. PLoS One 8(5):e62953 